Article

\title{
A Multi-Target Approach toward the Development of Novel Candidates for Antidermatophytic Activity: Ultrastructural Evidence on $\alpha$-Bisabolol-Treated Microsporum gypseum
}

\section{Carlo Romagnoli ${ }^{1}$, Anna Baldisserotto ${ }^{2}$, Gemma Malisardi ${ }^{2}$, Chiara B. Vicentini ${ }^{2}$, Donatella Mares ${ }^{2}$, Elisa Andreotti ${ }^{1}$, Silvia Vertuani ${ }^{2}$ and Stefano Manfredini ${ }^{2, *}$}

1 Department of Life Sciences, University of Modena and Reggio Emilia, viale Caduti in Guerra 127, 41121 Modena, Italy; E-Mails: carlo.romagnoli@unimore.it (C.R.); elisa.andreotti@unimore.it (E.A.)

2 Department of Life Sciences and Biotechnology, Master Course in Cosmetic Science and Technology, University of Ferrara, via Fossato di Mortara 17-19, 44121 Ferrara, Italy;

E-Mails: bldnna@unife.it (A.B.); gemma.malisardi@unife.it (G.M.); chiara.vicentini@unife.it (C.B.V.); donatella.mares@unife.it (D.M.); silvia.vertuani@unife.it (S.V.)

* Author to whom correspondence should be addressed; E-Mail: mv9@unife.it; Tel.: +39-0532-455294; Fax: +39-0532-455378.

Academic Editor: Derek J. McPhee

Received: 8 May 2015 / Accepted: 24 June 2015 / Published: 26 June 2015

\begin{abstract}
Multi-target strategies are directed toward targets that are unrelated (or distantly related) and can create opportunities to address different pathologies. The antidermatophytic activities of nine natural skin lighteners: $\alpha$-bisabolol, kojic acid, $\beta$-arbutin, azelaic acid, hydroquinone, nicotinamide, glycine, glutathione and ascorbyl tetraisopalmitate, were evaluated, in comparison with the known antifungal drug fluconazole, on nine dermatophytes responsible for the most common dermatomycoses: Microsporum gypseum, Microsporum canis, Trichophyton violaceum, Nannizzia cajetani, Trichophyton mentagrophytes, Epidermophyton floccosum, Arthroderma gypseum, Trichophyton rubrum and Trichophyton tonsurans. $\alpha$-Bisabolol showed the best antifungal activity against all fungi and in particular; against M. gypseum. Further investigations were conducted on this fungus to evaluate the inhibition of spore germination and morphological changes induced by $\alpha$-bisabolol by TEM.
\end{abstract}

Keywords: antifungal activity; dermatophytes; M. gypseum; $\alpha$-bisabolol; TEM; skin lightener 


\section{Introduction}

The skin is our first line of defense against the outside world. As the body's border, it is subject to various microbiological attacks. In particular, approximately $25 \%$ of the world's population suffers from skin mycosis [1], which represents one of the main causes of morbidity and mortality [2] and the seriousness of the disease is steadily increasing. Dermatophytoses are very fastidious and difficult to manage, and their therapy is complex and associated with high costs. Our research group has long been involved in the search for new molecules with antifungal activity, exploring not only the fields of unknown natural [3-6] and synthetic [7-10] substances but also those substances already used in other clinical fields. Our main interest is in the discovery of molecules with multiple activities (multi-target-drugs), the advantages from such an approach include not only a better balance between activity and side effects but also a reduction in cost and time to develop and reach the market. If multi-target strategies are directed toward targets that are unrelated (or distantly related) an opportunity maybe created to target different pathologies. In these regards, it is well known that skin hyperpigmentation is a relevant problem, affects a large portion of the world's population, and is a particular social issue for black and elderly people. This context has prompted the search for more effective treatments in dermatology and this problem is attributed to the over-accumulation of melanin in skin cells. Several molecules, mostly of natural origin, have been used over the last few years to counteract this effect. While the mechanisms of action are different, these molecules reduce melanin content. In a completely different field, melanin biosynthesis inhibitors have long been used for the control of plant pathogenic fungi $[9,11,12]$. We began an explorative investigation of these known melanin inhibitors, which may be used as antifungal agents in combination with known or investigational therapeutics. Agents that are typically used for dermatological purposes (e.g., skin lighteners), whose low toxicity, pharmacokinetics and pharmacodynamics are already known, will accelerate the process of developing innovative, less toxic and safer drug candidates. In particular, $\alpha$-bisabolol, kojic acid, $\beta$-arbutin, azelaic acid, hydroquinone, nicotinamide, glycine, glutathione and ascorbyl tetraisopalmitate were selected. These nine molecules are already known in the literature for their action as skin lighteners [13-17] and are present in several commercial products.

In this work, the antidermatophytic activity of these substances was tested on nine dermatophytes responsible for the most common dermatomycoses: Microsporum gypseum, Microsporum canis, Trichophyton violaceum, Nannizzia cajetani, Trichophyton mentagrophytes, Epidermophyton floccosum, Arthroderma gypseum, Trichophyton rubrum and Trichophyton tonsurans.

After a preliminary investigation, $\alpha$-bisabolol emerged as the most active compound; thus, the following experiments focused on this molecule and its effects on the most sensitive of the nine fungi, M. gypseum. This molecule (Figure 1) is a sesquiterpene alcohol found in the essential oils of several plants, such as Arnica longifolia, Aster esperius, Chrysothamnus nauseosus [18], and Chamomilla sp. [19], and its antibacterial and antifungal activities are well-known in the literature [20]. In particular, investigations were conducted by TEM to confirm whether the mechanism of action for the inhibition of ergosterol synthesis by $\alpha$-bisabolol, as suggested by Pauli [19] for other fungi, was also responsible for its antifungal activity [21]. 


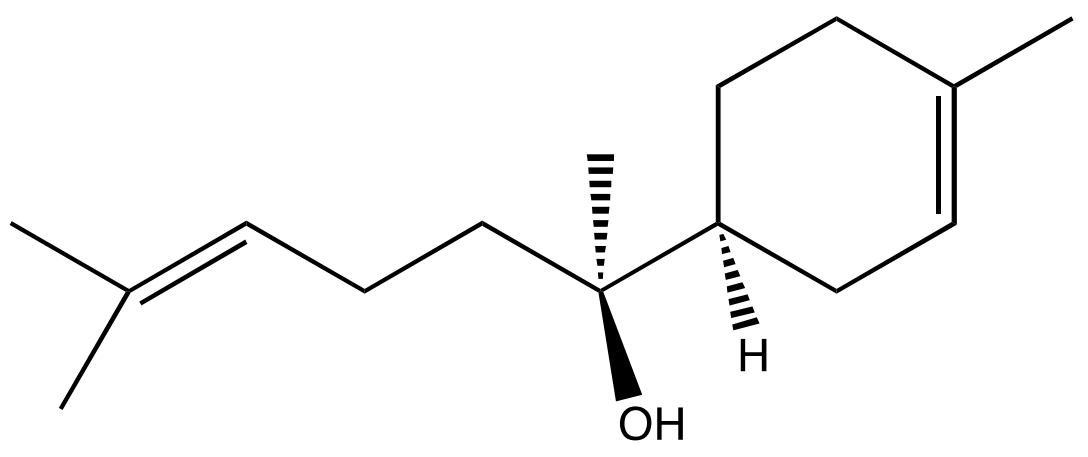

Figure 1. Chemical structure of $\alpha$-bisabolol.

\section{Results and Discussion}

\subsection{Antifungal Activity}

The growth inhibition data are shown in Table 1.

Table 1. Percent growth inhibition of dermatophytes treated with nine skin lighteners at 100 or $200 \mu \mathrm{g} / \mathrm{mL}$. Each value is the mean of three measurements.

\begin{tabular}{|c|c|c|c|c|c|c|c|c|c|c|}
\hline & & Kojic Acid & $\beta$-Arbutin & $\begin{array}{c}\text { Azelaic } \\
\text { Acid }\end{array}$ & Hydroquinone & Nicotinamide & $\alpha$-Bisabolol & Glycine & Glutathione & $\begin{array}{c}\text { Ascorbyl } \\
\text { TIP }\end{array}$ \\
\hline \multirow{2}{*}{ M. gypseum } & 100 & $3.5 \pm 0.1$ & $7.9 \pm 0.2$ & $6.9 \pm 0.2$ & $17.1 \pm 0.1$ & $9.8 \pm 0.1$ & $79.8 \pm 0.4$ & + & + & $16.6 \pm 0.3$ \\
\hline & 200 & $7.5 \pm 0.2$ & $27.9 \pm 0.1$ & $19.8 \pm 0.1$ & $51.2 \pm 0.1$ & $18.3 \pm 0.4$ & $83.7 \pm 0.2$ & $0.8 \pm 0.1$ & + & $21.3 \pm 0.2$ \\
\hline \multirow{2}{*}{ M. canis } & 100 & $22.6 \pm 0.3$ & $-11.8 \pm 0.2$ & $-5.9 \pm 0.1$ & $20.0 \pm 0.1$ & $2.4 \pm 0.1$ & $34.7 \pm 0.2$ & 0.0 & 0.0 & + \\
\hline & 200 & $29.0 \pm 0.1$ & $-2.9 \pm 0.2$ & $5.9 \pm 0.3$ & $46.7 \pm 0.2$ & $12.0 \pm 0.1$ & $43.1 \pm 0.2$ & + & $3.8 \pm 0.3$ & + \\
\hline \multirow{2}{*}{ T. violaceum } & 100 & $18.2 \pm 0.2$ & $-2.8 \pm 1.4$ & $-8.2 \pm 0.9$ & $20.0 \pm 1.2$ & $3.4 \pm 1.8$ & $31.0 \pm 0.8$ & + & $9.8 \pm 2.3$ & + \\
\hline & 200 & $19.9 \pm 1.1$ & $-5.0 \pm 0.7$ & $2.5 \pm 1.6$ & $30.6 \pm 1.3$ & + & $38.0 \pm 1.8$ & 0.0 & $12.0 \pm 1.4$ & $15.6 \pm 2.0$ \\
\hline \multirow{2}{*}{ N. cajetani } & 100 & $2.7 \pm 1.1$ & $6.9 \pm 1.6$ & $0.9 \pm 1.9$ & 0.0 & $14.4 \pm 0.7$ & $60.1 \pm 1.3$ & 0.0 & + & + \\
\hline & 200 & $8.0 \pm 1.0$ & $7.6 \pm 1.4$ & 0.0 & $4.2 \pm 0.3$ & $25.1 \pm 0.6$ & $62.7 \pm 1.5$ & + & $5.2 \pm 1.3$ & 0.0 \\
\hline \multirow{2}{*}{ T. mentagroph } & 100 & 0.0 & $-1.5 \pm 0.5$ & 0.0 & $29.7 \pm 1.8$ & $50.8 \pm 1.8$ & $67.0 \pm 1.3$ & $6.1 \pm 0.6$ & + & $9.8 \pm 1.1$ \\
\hline & 200 & $6.3 \pm 1.2$ & 0.0 & $9.3 \pm 0.8$ & $59.4 \pm 1.4$ & $78.0 \pm 3.1$ & $70.9 \pm 1.7$ & + & + & $19.6 \pm 0.8$ \\
\hline \multirow{2}{*}{ E. floccosum } & 100 & $1.9 \pm 1.7$ & $-1.6 \pm 0.5$ & $0.3 \pm 0.1$ & $2.5 \pm 0.2$ & $7.8 \pm 0.7$ & $31.8 \pm 1.1$ & + & $0.6 \pm 0.2$ & + \\
\hline & 200 & $2.5 \pm 0.9$ & $-1.3 \pm 0.5$ & $3.9 \pm 1.0$ & $5.4 \pm 0.8$ & + & $33.1 \pm 1.9$ & + & 0.0 & $6.4 \pm 0.9$ \\
\hline \multirow{2}{*}{ A. gypseum } & 100 & $10.0 \pm 1.2$ & 0.0 & $7.4 \pm 1.4$ & $-11.0 \pm 0.9$ & $1.9 \pm 1.2$ & $62.0 \pm 1.7$ & + & $0.6 \pm 2.1$ & $0.7 \pm 0.5$ \\
\hline & 200 & $11.3 \pm 1.2$ & $2.3 \pm 1.8$ & $3.7 \pm 1.4$ & $2.4 \pm 1.1$ & $7.1 \pm 0.6$ & $65.8 \pm 1.3$ & + & $1.3 \pm 1.0$ & + \\
\hline \multirow{2}{*}{ T. rubrum } & 100 & $-0.4 \pm 0.8$ & $6.1 \pm 1.4$ & $0.8 \pm 0.5$ & $-2.3 \pm 1.9$ & $4.5 \pm 0.3$ & $14.6 \pm 1.9$ & + & $2.6 \pm 0.2$ & 0.0 \\
\hline & 200 & $2.9 \pm 0.7$ & $6.8 \pm 1.3$ & $1.6 \pm 0.7$ & $2.9 \pm 0.6$ & $10.8 \pm 1.1$ & $37.4 \pm 1.4$ & + & + & + \\
\hline \multirow{2}{*}{ T. tonsurans } & 100 & 0.0 & $4.4 \pm 0.5$ & $-2.6 \pm 1.2$ & $40.0 \pm 1.1$ & $9.3 \pm 1.3$ & $69.6 \pm 1.9$ & + & + & + \\
\hline & 200 & $5.0 \pm 0.5$ & $11.1 \pm 0.7$ & 0.0 & $55.00 \pm 1.5$ & + & $71.7 \pm 1.2$ & $0.0 \pm 0.8$ & + & + \\
\hline
\end{tabular}

In general, the nine substances showed low inhibition of growth. In fact, after treatment with $\beta$-arbutin, increased fungal growth was observed for $M$. canis and $T$. violaceum. The only substances that showed significant inhibition values were hydroquinone and $\alpha$-bisabolol. The former showed inhibition values greater than $50 \%$ at the highest dose $(200 \mu \mathrm{g} / \mathrm{mL})$ for $M$. gypseum $(51 \%)$, T. tonsurans (55\%) and T. mentagrophytes (59.5\%). At the lower dose $(100 \mu \mathrm{g} / \mathrm{mL})$, a $40 \%$ inhibition value was observed for $T$. tonsurans. When treated with $100 \mu \mathrm{g} / \mathrm{mL} \alpha$-bisabolol, at least $30 \%$ inhibition was observed 
for all fungi, with the exception of T. rubrum. For both the lower and higher doses of $\alpha$-bisabolol, inhibition was greater than $50 \%$ for five fungi (M. gypseum, N. cajetani, T. mentagrophytes, T. tonsurans and $A$. gypseum). In particular, T. mentagrophytes and $T$. tonsurans were sensitive to treatment, reaching approximately $70 \%$ inhibition for both dose levels. For treatments with $100 \mu \mathrm{g} / \mathrm{mL}$ and $200 \mu \mathrm{g} / \mathrm{mL}$ of $\alpha$-bisabolol, the highest inhibition levels were observed in M. gypseum at $79.8 \%$ and $83.7 \%$, respectively. Due to this high inhibition activity, we chose M. gypseum for further studies at the ultrastructural level to determine morphological changes induced by $\alpha$-bisabolol.

In Table 2 are shown the $\mathrm{IC}_{50}$ values of $\alpha$-bisabolol, in comparison with the well known antifungal agent, fluconazole.

Table 2. IC50 values of $\alpha$-bisabolol and Fluconazole on dermatophytes. Each value is the mean of three measurements.

\begin{tabular}{lcc}
\hline & a-Bisabolol & Fluconazole \\
\cline { 2 - 3 } & \multicolumn{2}{c}{$\mathbf{I C}_{\mathbf{5 0}} \boldsymbol{\mu \mathrm { g } / \mathbf { m L }}$} \\
\hline M. gypseum & $35.24 \pm 0.9$ & $18.5 \pm 0.2$ \\
M. canis & $>200$ & $29.6 \pm 0.3$ \\
T. violaceum & $>200$ & $31.03 \pm 0.9$ \\
N. cajetani & $85 \pm 0.6$ & $>200$ \\
T. mentagrophytes & $60.51 \pm 0.3$ & $3.53 \pm 0.8$ \\
E. floccosum & $>200$ & $0.08 \pm 0.6$ \\
A. gypseum & $81.69 \pm 0.4$ & $>200$ \\
$T$. rubrum & $>200$ & $37.16 \pm 0.6$ \\
T. tonsurans & $49.38 \pm 0.7$ & $19.41 \pm 0.5$ \\
\hline
\end{tabular}

$\mathrm{IC}_{50}$ values confirmed the interesting activity of $\alpha$-bisabolol, in particular on $M$. gypseum $(35.24 \mu \mathrm{g} / \mathrm{mL})$ and $T$. tonsurans $(49,38 \mu \mathrm{g} / \mathrm{mL})$, it is remarkable that on two fungi (N. cajetani and A. gypseum) the $\mathrm{IC}_{50}$ was even lower than that of fluconazole.

\subsection{Effect of Time and Spore Density on Percent Reduction of Resazurin}

The effect of spore density and incubation time on reduction of Resazurin, are illustrated in Figure 2. The blue color indicates absence of cell viability while the pink color indicates the presence of cell viability. It can be seen that the first signs of cell viability appeared at a density of $10^{5} \mathrm{spores} / \mathrm{mL}$ and after an incubation time at $24{ }^{\circ} \mathrm{C}$ of $120 \mathrm{~h}$. We considered these values of spore density and incubation time the best for the evaluation of inhibition of spore germination.

\subsection{Inhibition of Spore Germination by Resazurin Assay}

Table 3 presents the values of percent inhibition rate of spore germination with $\alpha$-bisabolol at the concentrations of 100 and $200 \mu \mathrm{g} / \mathrm{mL}$. It appeared to be a good inhibitor of spore germination at both tested doses of 100 and $200 \mu \mathrm{g} / \mathrm{mL}$ (52.08\% and 59.32\% inhibition respectively). It is noteworthy that these small differences in inhibition of spore germination corresponded well with the effect of $\alpha$-bisabolol on growth at both doses (79.8\% and $83.7 \%$ inhibition respectively). 

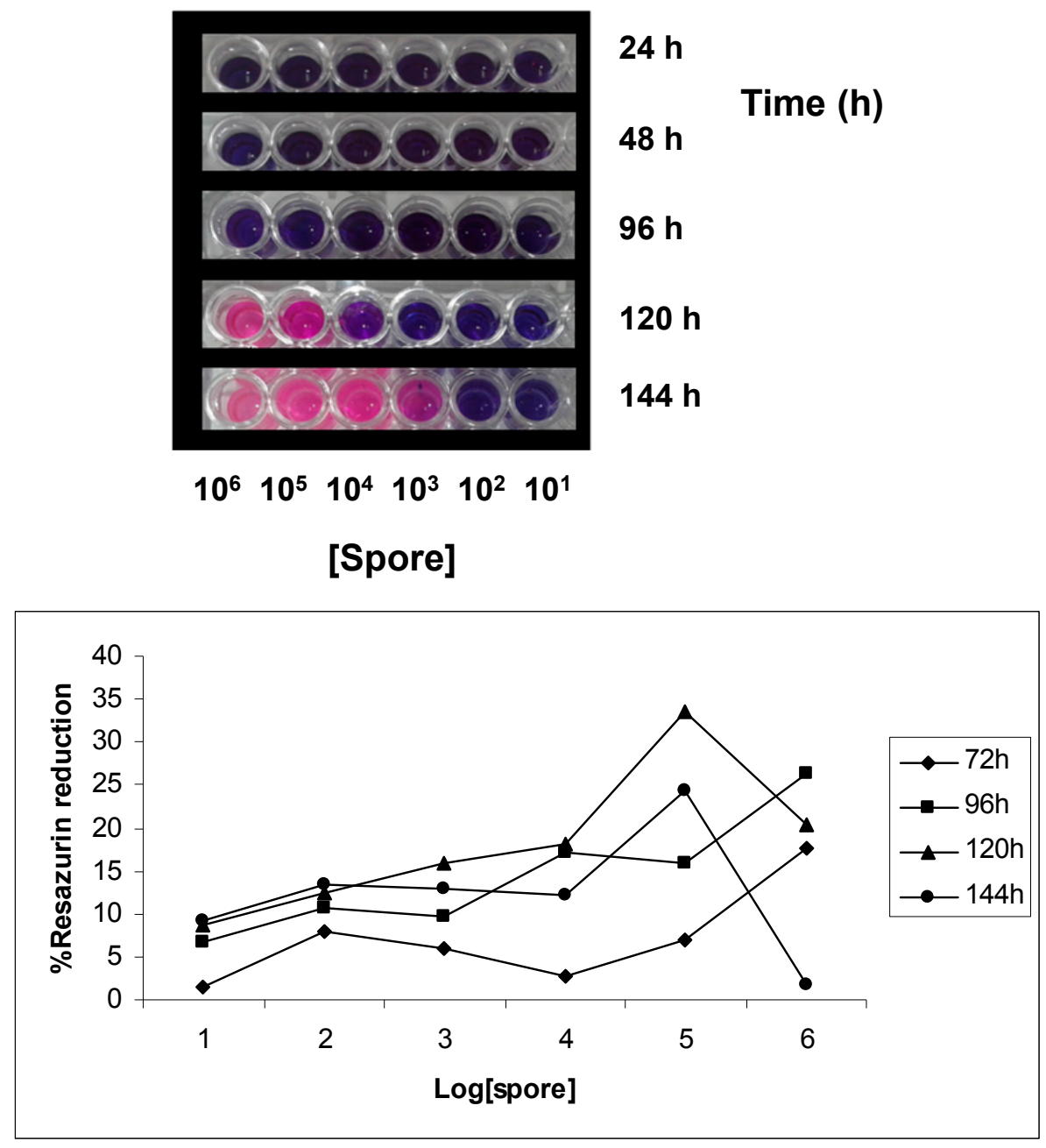

Figure 2. Effect of time and spore density on percent reduction of Resazurin. The highest reduction occurred after $120 \mathrm{~h}$ of incubation with a spore density of $10^{5}$ spores.

Table 3. Percent inhibition values on spore germination.

\begin{tabular}{cc}
\hline$\alpha$-Bisabolol Concentration & \%Inhibition of Spore Germination \\
\hline $100 \mu \mathrm{g} / \mathrm{mL}$ & $52.08 \pm 0.9$ \\
$200 \mu \mathrm{g} / \mathrm{mL}$ & $59.32 \pm 1.3$ \\
\hline
\end{tabular}

\subsection{TEM}

When examined by TEM, the mycelium of the M. gypseum control sample appeared normal: all organelles, such as the nucleus and mitochondria, were normally shaped, and the plasma membrane was unfolded with a uniform appearance (Figure 3A).

After $24 \mathrm{~h}$ of treatment at the lower $\alpha$-bisabolol concentration of $100 \mu \mathrm{g} / \mathrm{mL}$, morphological changes were observed. As shown in Figure 3B, the nuclei are irregularly shaped, presenting numerous anomalous lobes and invaginations; even the nucleoli appear frayed and not very compact.

In Figure 4A, after treatment at the higher $200 \mu \mathrm{g} / \mathrm{mL}$ concentration, the outer cell wall presents significant abnormalities. In particular, the anomalous formation of numerous and recurring septa are visible; the septa often have altered shapes, such as the bifid septum indicated by the arrow. In addition, the septa are located in unusual areas. In Figure 4B, there is evidence of the early formation of a septum 
in the sub apical area, which is a location where parietal components are still in phase of linkage. In the same figure, a thick cell wall (indicated by an asterisk) is present in an adjacent cell, made of many layers and it is also visible an abnormal extrusion of cell wall materials from the outer layer.

Finally, increased vacuolization is observed within the cytoplasm after treatment with $200 \mu \mathrm{g} / \mathrm{mL}$ of $\alpha$-bisabolol.
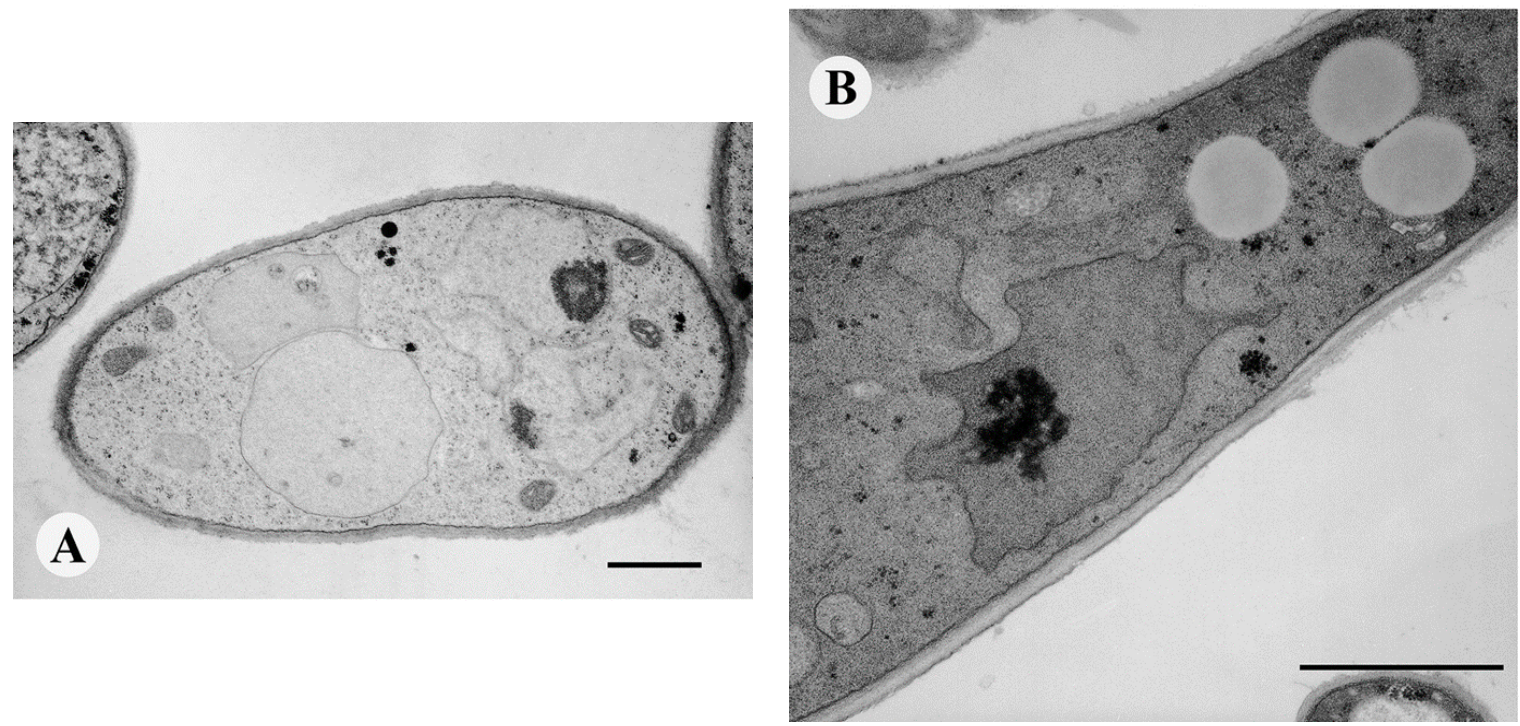

Figure 3. (A) Untreated control hypha of M. gypseum observed by TEM. The nucleus and the cell wall are normally structured; (B) TEM of M. gypseum treated with $100 \mu \mathrm{g} / \mathrm{mL}$ $\alpha$-bisabolol. The nucleus shows deep invaginations. Bar, $1 \mu \mathrm{m}$.
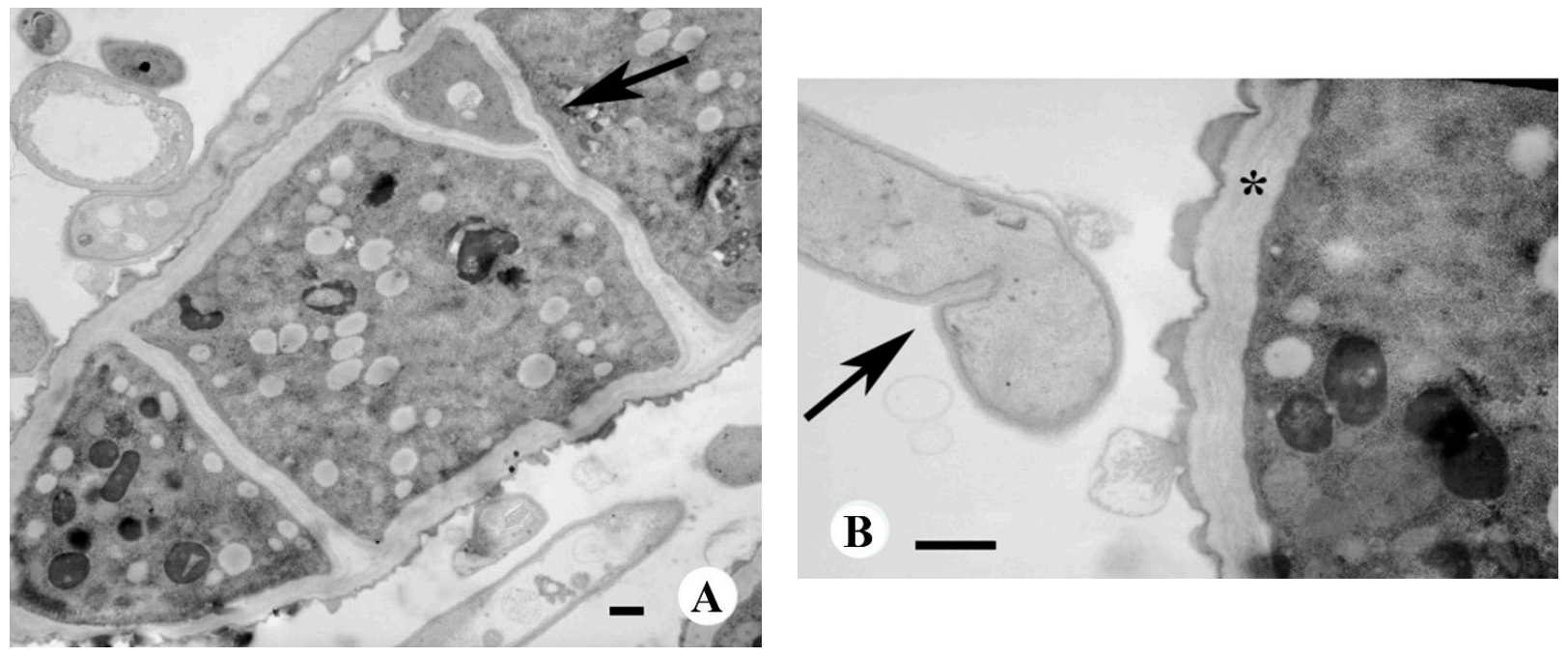

Figure 4. TEM of M. gypseum treated with $200 \mu \mathrm{g} / \mathrm{mL} \alpha$-bisabolol. (A) Septa are in closer proximity with each other and have anomalous shapes; arrow indicates a bifidous septum; (B) Arrow indicates the early formation of a septum in the sub apical area; asterisk indicates an abnormal multilayer cell wall. Bar, $1 \mu \mathrm{m}$.

Our study shows that, among the nine skin lighteners tested as antifungal agents, $\alpha$-bisabolol showed promising properties, due to its ability to inhibit the growth of all tested dermatophytes, especially M. gypseum. The $\mathrm{IC}_{50}$ of $\alpha$-bisabolol on $M$. gypseum suggested us to choose this fungus to better 
understand the antidermatophytic activity. The first step was the evaluation of spore germination inhibition with the Resazurin assay. This is a widespread test in agronomy, but only little applied on dermatophytes and never used on M. gypseum. So it was necessary to adapt the procedure, by determing the right number of spore and the time of germination of this fungus. The results obtained with Resazurin assay on spore germination, confirmed the good inhibition activity of $\alpha$-bisabolol on M. gypseum.

TEM observations were performed on an $\alpha$-bisabolol-treated $M$. gypseum sample to better understand the mechanism of action of this anti-fungal agent. Upon treatment with $\alpha$-bisabolol, several morphological abnormalities were observed in M. gypseum. At a low $\alpha$-bisabolol concentration, the nuclear envelope presented numerous lobes, whereas at a higher dose, the aberrations are widespread, even affecting the cell wall structure. Both aspects are likely related to altered microtubule behavior. $\alpha$-Bisabolol may affect the dynamic microtubules responsible for maintaining the nuclear shape, causing alterations in the nuclear envelope [22,23]. Additionally, it is also possible that $\alpha$-bisabolol targets the microtubules responsible for directing cellular wall materials toward the cell periphery. It is known that MTs and other cytoskeleton components are responsible for the normal assembly of parietal components and are involved in the transport and release of vesicles containing the construction materials [24]. Disruption of the MTs leads to altered vesicle distributions [25] and thus results in the irregular build-up of wall materials to apical locations along the cell wall. This proposed mechanism could also explain the anomalous septa appearances in the apical zone and the increased concentration of septa. The observed effects correspond with the effects of $\alpha$-bisabolol when used as a biosynthetic inhibitor of ergosterol, a sterol specifically located in the membrane of fungi, as suggested by Pauli (2006). In this previous study, by comparing the chemical structures of $\alpha$-bisabolol and zymosterol, Pauli suggested treatment with $\alpha$-bisabolol prevented the formation of fecosterol from zymosterol, which is the first fungi-specific step in ergosterol biosynthesis. Thus, $\alpha$-bisabolol has a mechanism of action common to several antifungal drugs [26,27].

$\alpha$-Bisabolol may exists as four different isomers. The natural, pure isomer is the RR (-) enantiomer, which can only be extracted from natural origins. While the $(-)$ and $(+)$ racemic mixtures of $\alpha$-bisabolol can be chemically synthesized in the laboratory, the separation of the two enantiomers is economically disadvantageous. The racemic mixtures have half the effective biological activity of the pure, naturally derived (-)a-bisabolol.

\section{Experimental Section}

\subsection{Chemicals}

The tested substances ( $\alpha$-bisabolol, kojic acid, $\beta$-arbutin, azelaic acid, hydroquinone, nicotinamide, glycine, glutathione and ascorbyl tetraisopalmitate) were purchased from Fagron, API Corporation, Res Pharma, Sarfam Comercial Importadora Ltda. (Sao Paolo, SP, Brazil) and Nikko Chemicals Co., Ltd. (Tokyo, Japan).

Fungal culture medium was purchased from Difco Laboratories, Inc. (Becton, Dickinson and Company, Cockeysville, MD, USA).

The chemicals used for fixations and solvents were purchased from Sigma-Aldrich SRL, Milano, Italy. 


\subsection{Microorganisms}

Seven of the specific fungi strains investigated in this study were purchased from the Centraal Bureau voor Schimmelcultures (CBS), Baarn, The Netherlands: Nannizzia cajetani Ajello, strain CBS 495.70; Epidermophyton floccosum (Hartz) Langeron and Milochevitch, strain CBS 358.93; Trichophyton violaceum Malmsten, strain CBS 459.61; Trichophyton tonsurans Malmsten, strain CBS 483.76, Trichophyton mentagrophytes (Robin) Blanchard, strain CBS 160.66; Microsporum canis Bodin, strain CBS 4727; and Arthroderma gypseum (Nann.) Weitzman, McGinnis, A.A. Padhye and Ajello, strain CBS 286.63. The remaining two strains were purchased from the Institute of Hygiene and Epidemiology-Mycology Laboratory (IHME), Brussels, Belgium: Trichophyton rubrum (Castellani) Sabouraud, strain IHME 4321; Microsporum gypseum (Bodin) Guiart e Grigorakis, strain IHME 3999. All dermatophytes were maintained at $4{ }^{\circ} \mathrm{C}$ as agar slants on Sabouraud dextrose agar (SDA; Difco Laboratories, Inc.).

\subsection{Antifungal Activity}

Antifungal activity was determined as follows. Each test substance was dissolved in dimethylsulfoxide (DMSO) and aseptically mixed with sterile medium (SDA) at $45{ }^{\circ} \mathrm{C}$ to concentrations of 100 and $200 \mu \mathrm{g} / \mathrm{mL}$. The DMSO concentration in the final solution was adjusted to $0.1 \%$. Controls were also prepared with equivalent concentrations $(0.1 \% v / v)$ of DMSO. For experiments, cultures were obtained by transplanting mycelium disks $(10 \mathrm{~mm}$ diameters $)$ from a single mother culture in the stationary phase. They were incubated at $26 \pm 1{ }^{\circ} \mathrm{C}$ on SDA on thin sheets of cellophane until the logarithmic growth phase. Subsequently, the cultures were transferred to Petri plates with media containing 100 or $200 \mu \mathrm{g} / \mathrm{mL}$ of the single substance and incubated under growth conditions. The fungal growth was evaluated daily by measuring colony diameters (in millimeters) for seven days from the treatment onset.

The percent inhibition of growth was determined as the average of three different experiments.

$\mathrm{IC}_{50}$ values were obtained testing $\alpha$-bisabolol and Fluconazole against the nine dermatophytes at different concentrations: 1, 5, 10, 20, $50 \mu \mathrm{g} / \mathrm{mL}$ for Fluconazole, 5, 10, 20, 50, $100 \mu \mathrm{g} / \mathrm{mL}$ for $\alpha$-bisabolol.

$\mathrm{IC}_{50}$ values were determined as the average of three different experiments.

\subsection{Determination of Optimal Spore Concentration-Growth in Sabouraud Dextrose Broth in Resazurin Assay}

M. gypseum was grown on Sabouraud Dextrose (SD) agar at $28{ }^{\circ} \mathrm{C}$ until sporulation occurs, typically for 7-14 days. The spores were harvested in Sabouraud Dextrose (SD) agar from 12 days cultures and the numbers of Colony Forming Units (CFU) per $\mathrm{mL}$ were determined by plating serial dilutions on Sabouraud Dextrose agar plates.

Subsequently, serial dilutions were made from a first solution containing $6 \times 10^{6}$ spores $/ \mathrm{mL}$ in Sabouraud Dextrose Broth to obtain spore concentrations from $10^{1}$ to $10^{6}$ spores $/ \mathrm{mL}$. The vials were prepared in duplicates for each concentration. Then, $100 \mu \mathrm{L}$ of a stock solution of Resazurin (Resazurin sodium salt, Sigma, stock solution of $0.00675 \mathrm{~g} / \mathrm{mL}$ of sterile distilled water) was added to test vials. Vials were covered, gently rotated horizontally to mix the vial contents, and incubated in the dark at $24{ }^{\circ} \mathrm{C}$. At six different incubation times $(24,48,72,96,120$ and $144 \mathrm{~h})$ the percent reduction of Resazurin 
was determined by taking absorbance readings at two wavelengths, 540 and $630 \mathrm{~nm}$. Duplicate negative control vials, contained $10 \mathrm{~mL}$ of medium and $100 \mu \mathrm{L}$ of Resazurin only, were made.

\subsection{Evaluation of Inhibition of Spore Germination of $\alpha$-Bisabolol}

The efficacy of $\alpha$-bisabolol was evaluated by the Resazurin assay using optimized incubation time and spore density. A stock solution of $\alpha$-bisabolol was prepared in DMSO. Then $\alpha$-bisabolol was added to test vials in duplicate at the concentrations of 20 and $100 \mu \mathrm{g} / \mathrm{mL}$. Test vials contained $\alpha$-bisabolol, $10^{5}$ spore $/ \mathrm{mL}$ (determined from previous evaluation), and $100 \mu \mathrm{L}$ of Resazurin stock solution Sabouraud Dextrose Broth. The vials were covered, gently rotated horizontally to mix the content, and incubated in the dark at $24{ }^{\circ} \mathrm{C}$ for $120 \mathrm{~h}$. Duplicate negative control vials contain $10 \mathrm{~mL}$ of medium and $100 \mu \mathrm{L}$ of Resazurin stock solution. Duplicate positive control vials containe $10 \mathrm{~mL}$ of medium, $10^{5}$ spore $/ \mathrm{mL}$, and $100 \mu \mathrm{L}$ of Resazurin stock solution.

\subsection{Spectrophotometric Measurement and Visual Inspection}

Absorbance data was expressed as percent Resazurin reduced as a function of incubation time. After $120 \mathrm{~h}$, the percent reduction of Resazurin was determined colorimetrically by taking absorbance readings at two wavelengths, 540 and $630 \mathrm{~nm}$, to compensate for an overlap in the absorption spectra of the oxidized and reduced forms of the dye [28]). Absorbance was read with a Beckman Coulter TM $D^{\circledR}$ 530, Life Science UV/Vis Spectrophotometer Single cell module. The mean absorbance values were used in subsequent calculations. The following Equation (1) was used to calculate the percent reduction of Resazurin:

Percent reduction of Resazurin $=[(\mathrm{O} 2 \times \mathrm{A} 1)-(\mathrm{O} 1 \times \mathrm{A} 2)] /[(\mathrm{R} 1 \times \mathrm{N} 2)-(\mathrm{R} 2 \times \mathrm{N} 1)] \times 100$

where $\mathrm{O} 1=$ molar extinction coefficient $(\mathrm{E})$ of oxidized Resazurin (Blue) at $540 \mathrm{~nm}, \mathrm{O} 2=\mathrm{E}$ of oxidized Resazurin at $630 \mathrm{~nm}, \mathrm{R} 1=\mathrm{E}$ of reduced Resazurin (Red) at $540 \mathrm{~nm}, \mathrm{R} 2=\mathrm{E}$ of reduced Resazurin at $630 \mathrm{~nm}, \mathrm{~A} 1=$ absorbance of test vials at $540 \mathrm{~nm}, \mathrm{~A} 2=$ absorbance of test wells at $630 \mathrm{~nm}, \mathrm{~N} 1$ = absorbance of negative control vial (media plus Resazurin but no cells) at $540 \mathrm{~nm}$, and $\mathrm{N} 2$ = absorbance of negative control vial (media plus Resazurin but no cells) at $630 \mathrm{~nm}$. Molar extinction coefficients were $540 \mathrm{~nm}=104,395$, reduced and 47,619, oxidized; and $630 \mathrm{~nm}=5494$, reduced and 34,798, oxidized.

A blue color was interpreted as absence of metabolic activity (no spore germination). A fluorescent pink color was interpreted as presence of metabolic activity (spore germination). A purple color in the vials was interpreted as a trailing result where some metabolic activities were present but a longer incubation time allowed the purple color to change to pink.

\subsection{Transmission Electron Microscopy (TEM)}

For TEM, the youngest hyphae of $M$. gypseum were chosen from untreated mycelia and from mycelia treated for $24 \mathrm{~h}$ with 100 and $200 \mu \mathrm{g} / \mathrm{mL}$ of $\alpha$-bisabolol.

The samples were fixed with $6 \%$ glutaraldehyde (GA) in a $0.1 \mathrm{M}$ sodium cacodylate buffer, $\mathrm{pH} 6.8$ for $6 \mathrm{~h}$ at $4{ }^{\circ} \mathrm{C}$. After rinsing in the same buffer solution, they were post-fixed for $15 \mathrm{~h}$ with $1 \%$ osmium tetroxide $\left(\mathrm{OsO}_{4}\right)$ in the same buffer, dehydrated in a graded series of ethanol solutions and embedded 
in Epon-Araldite resin. Sections were cut with an LKB Ultratome III, stained with uranyl acetate and lead citrate and observed under a Hitachi H-800 electron microscope at $100 \mathrm{kV}$ (provided by the Electron Microscopy Center of Ferrara University).

\section{Conclusions}

In conclusion, we have shown that $\alpha$-bisabolol, a natural product present in many preparations intended for cosmetic and dermatological uses, possesses significant antifungal activity in vitro, against dermatophytes. This property suggests its applicability, within a multidrug approach, for the treatment of dermatomycoses, which are especially persistent diseases in immunocompromised hosts [20]. Ongoing studies are now evaluating the synergistic combination of $\alpha$-bisabolol with traditional antifungal agents for the treatment of dermatophytoses, and antifungal activity against other pathogenic fungi. Although many antifungals are available, their side effects and drug interactions, and the existence of resistant organisms have created a need to find safer and more effective treatments. Cytotoxicity assay will be performed to assess the selectivity of the compound in the same conditions as the antifungal test.

\section{Acknowledgments}

We wish to thank Bovolenta and the staff of the Electron Microscopy Center of Ferrara University for their skillful technical assistance. We also wish to thank Riccardo Marcato (Department of Land, Environment, Agriculture and Forestry TESAF, of the University of Padova), for his valuable contribution in resazurin assay. S.M., C.B.V. and S.V. thank the Ministry of Education and Research (PRIN, Grant 20082L3NFT_003) and Ambrosialab srl (Ferrara, Italy) for financial support.

\section{Author Contributions}

S.M. proposed the subject; S.M. and C.R. designed and supervised the study; A.B., G.M. and E.A. carried out production and analysis; C.B.V, D.M. and S.V. contributed to the interpretation of the results; C.R., A.B., S.V. and S.M. wrote the manuscript. All the authors read and approved the final manuscript.

\section{Conflicts of Interest}

The authors declare no conflict of interest.

\section{References}

1. Havlickova, B.; Czaika, V.A.; Friedrich, M. Epidemiological trends in skin mycoses worldwide. Mycoses 2008, 51, 2-15.

2. Jamalian, A.; Shams-Ghahfarokhi, M.; Jaimand, K.; Pashootan, N.; Amani, A.; Razzaghi-Abyaneh, M. Chemical composition and antifungal activity of Matricaria recutita flower essential oil against medically important dermatophytes and soil-borne pathogens. J. Mycol. Med. 2012, 22, 308-315.

3. Mares, D. Electron microscopy of Microsporum cookie after "in vitro" treatment with protoanemonin: A combined SEM and TEM study. Mycopathologia 1989, 108, 37-46. 
4. Romagnoli, C.; Mares, D.; Fasulo, M.P.; Bruni, A. Antifungal effects of $\alpha$-terthienyl from Tagetes patula on five dermatophytes. Phytother. Res. 1994, 8, 332-336.

5. Romagnoli, C.; Bruni, R.; Andreotti, E.; Rai, M.K.; Vicentini, C.B.; Mares, D. Chemical characterization and antifungal activity of essential oil of capitula from wild Indian Tagetes patula $\mathrm{L}$. Protoplasma 2005, 225, 57-65.

6. Mares, D.; Romagnoli, C.; Tosi, B.; Andreotti, E.; Chillemi, G.; Poli, F. Chicory extracts from Cichorium intybus L. as potential antifungals. Mycopathologia 2005, 160, 85-91.

7. Mares, D.; Romagnoli, C.; Sacchetti, G.; Vicentini, C.B.; Bruni, A. Morphological study of Trichophyton rubrum; ultrastructural findings after treatment with 4-amino-3-methyl-1phenylpyrazolo-(3,4-c)isothiazole. J. Mycol. Med. 1998, 36, 379-385.

8. Mares, D.; Romagnoli, C.; Tosi, B.; Benvegnù, R.; Bruni, A.; Vicentini, C.B. Mannan changes induced by 3-methyl-5-aminoisoxazole-4-thiocyanate, a new azole derivative, on Epidermophyton floccosum. Fungal Genet. Biol. 2002, 36, 47-57.

9. Vicentini, C.B.; Forlani, G.; Manfrini, M.; Romagnoli, C.; Mares, D. Development of New Fungicides against Magnaporthe grisea: Synthesis and Biological Activity of Pyrazolo[3,4-d][1,3]thiazine, Pyrazolo[1,5-c][1,3,5]thiadiazine, and Pyrazolo[3,4-d]pyrimidine Derivatives. J. Agric. Food Chem. 2002, 50, 4839-4845.

10. Vicentini, C.B.; Romagnoli, C.; Andreotti, E.; Mares, D. Synthetic Pyrazole Derivatives as Growth Inhibitors of Some Phytopathogenic Fungi. J. Agric. Food Chem. 2007, 55, 10331-10338.

11. Kurahashi, Y. Melanin biosynthesis inhibitors (MBIs) for control of rice blast. Pestic. Outlook 2001, 12, 32-35.

12. Mares, D.; Romagnoli, C.; Andreotti, E.; Forlani, G.; Guccione, S.; Vicentini, C.B. Emerging antifungal azoles and effects on Magnaporthe grisea. Mycol. Res. 2006, 110, 686-696.

13. Parvez, S.; Kang, M.; Chung, H.S.; Cho, C.; Hong, M.C.; Shin, M.K.; Bae, H. Survey and mechanism of skin depigmenting and lightening agents. Phytother. Res. 2006, 20, 921-934.

14. Alexis, A.F.; Blackcloud, P. Natural ingredients for darker skin types: Growing options for hyperpigmentation. J. Drugs Dermatol. 2013, 12, 123-127.

15. Ishikawa, M.; Kawas, I.; Ishii, F. Glycine inhibits melanogenesis in vitro and causes hypopigmentation in vivo. Biol. Pharm. Bull. 2007, 30, 2031-2036.

16. Lee, J.; Jun, H.; Jung, E.; Ha, J.; Park, D. Whitening effect of alpha-bisabolol in Asian women subjects. Int. J. Cosmet. Sci. 2010, 32, 299-303.

17. Solano, F.; Briganti, S.; Picardo, M.; Ghanem, G. Hypopigmenting agents: An updated review on biological, chemical and clinical aspects. Pigment Cell Res. 2006, 19, 550-571.

18. Tabanca, N.; Demirci, B.; Crockett, S.A.L.; Baser, K.H.C.; Wedge, D.E. Chemical Composition and Antifungal Activity of Arnica longifolia, Aster hesperius, and Chrysothamnus nauseosus Essential Oils. J. Agric. Food Chem. 2007, 55, 8430-8435.

19. Pauli, A. $\alpha$-Bisabolol from Chamomile - A specific ergosterol biosynthesis inhibitor? Int. J. Aromather. 2006, 16, 21-25.

20. De Lucca, A.; Pauli, A.; Schilcher, H.; Sien, T.; Bhatnagar, D.; Walsh, T.J. Fungicidal and bactericidal properties of bisabolol and dragosantol. J. Essent. Oil Res. 2011, 23, 47-54.

21. Tabassum, N.; Vidyasagar, G.M. Antifungal investigations on plant essential oils. Int. J. Pharm. Pharm. Sci. 2013, 5, 19-28. 
22. Inoue, S.; Turgeon, B.G.; Yoder, O.C.; Aist, J.R. Role of fungal dynein in hyphal growth, microtubule organization, spindle pole body motility and nuclear migration. J. Cell Sci. 1998, 111, 1555-1566.

23. Beaudouin, J.; Gerlich, D.; Eils, N.D.R.; Ellenberg, J. Nuclear Envelope Breakdown Proceeds by Microtubule-Induced Tearing of the Lamina. Cell 2002, 108, 83-96.

24. Mares, D. Fungal morphogenesis induced by natural and synthetic substances: Herniarin-induced alterations in the dermatophyte Microsporum cookie Ajello. Plant Biosyst. 2005, 139, 323-334.

25. Howard, R.J.; Aist, J.R. Cytoplasmic microtubules and fungal morphogenesis: Ultrastructural effects of methyl benzimidazole-2-yl carbamate determined by freeze substitution of hyphal cells. J. Cell Biol. 1980, 118, 109-120.

26. Georgopapadakou, N.H. Antifungals: Mechanism of action and resistance, established and novel drugs. Curr. Opin. Microbiol. 1998, 1, 547-557.

27. Odds, F.C.; Brown, A.J.P.; Gow, N.A.R. Antifungal agents: Mechanisms of action. Trends Microbiol. 2003, 11, 272-279.

28. Rampersad, S.N. A rapid colorimetric microtiter bioassay to evaluate fungicide sensitivity among Verticillum dahliae Isolates. Plant Dis. 2011, 95, 248-255.

Sample Availability: Samples of the compounds are available from the authors.

(C) 2015 by the authors; licensee MDPI, Basel, Switzerland. This article is an open access article distributed under the terms and conditions of the Creative Commons Attribution license (http://creativecommons.org/licenses/by/4.0/). 